\title{
OPTIMAL PLANE CHANGES IN TIDALLY PERTURBED ENVIRONMENTS *
}

\author{
B.F. Villac ${ }^{\dagger}$ and D.J. Scheeres ${ }^{\ddagger}$ \\ The University of Michigan \\ Ann Arbor, Michigan 48109-2140
}

\begin{abstract}
For planetary satellite orbiters (such as the Europa Orbiter) and planet orbiters (such as the Mercury Messenger Mission), tidal forces can induce large changes in orbital elements over one orbit. The longitude of the ascending node and the argument of periapsis of a transfer orbit can be viewed as control variables since they have a strong influence on the dynamics, and can be set to an arbitrary precision by proper timing. In this paper we investigate some aspects of the practical application of transfer maneuvers in a strongly perturbed tidal environment with a focus on plane change maneuvers. In particular, transfers involving an ellipse cannot be considered without taking the tidal forces into account, and two and three impulse transfers must be replaced by tidally driven transfers. These tidally driven plane changes are shown to be optimal over classical plane change maneuvers for plane changes larger than $\sim 38^{\circ}$, replacing the classical optimality of bi-elliptic transfers over the one impulse plane changes in the case of tidally perturbed environments. The investigation is performed by solving an optimization problem with constraints and using the Hill three body problem for the underlying dynamics. Savings on the order of $25 \%$ are obtained when compared to the one impulse transfers for plane changes of $\sim 60^{\circ}$ and plane changes of $-180^{\circ}$ are shown to be possible (with $\sim 15 \%$ savings).
\end{abstract}

\section{Introduction}

Planetary satellite orbiter missions, like the Europa Orbiter Mission, present new challenges to the design of trajectories. In such environments the tidal forces due to the nearby planet (Jupiter in the case of Europa) are

\footnotetext{
* Paper AIAA 2002-4724. Copyright (c)2002 by the authors.

$\dagger$ Ph.D. Candidate, Department of Aerospace Engineering

$\ddagger$ Senior Member AIAA, Member AAS, Associate Professor, Department of Aerospace Engineering.
}

strong enough to cause instabilities that can result in the impact of the orbiter on the satellite over relatively short time spans, even for low altitude trajectories. In particular, while low inclination orbits have been shown to be stable, scientifically interesting orbits, like near polar orbits needed for high latitude mapping of the satellite, have been shown to be unstable ${ }^{1}$. Assuming a spacecraft in such an unstable trajectory, large plane change maneuvers may then be required to transfer it into a lower inclination, stable orbit, in order to avoid impact with the satellite over long time spans. During the transfer the tidal perturbations are of prime concern since two and three impulse transfers involve raising the apoapsis radius, resulting in larger perturbations. As a consequence, classical types of transfers are not directly applicable to this environment as one must take these tidal perturbations into account. As shown in a previous paper ${ }^{2}$, these dynamics are strongly dependant on the orbit geometry at apoapsis and, in particular, on the longitude of the ascending node $\Omega$ and the argument of peripasis $\omega$. These variables allow us to use tidal perturbations as control forces for the design of transfer maneuvers, resulting in lower costs and new optimal criteria for these maneuvers.

In this paper, focus is given to the case of tidally driven plane change maneuvers, a problem initially motivated by the Europa Orbiter mission. The underlying dynamics are modeled using the Hill three body problem, which is a good model for the dynamics of particles in the environment of a planetary satellite (or even a Sun-planet system) and present attractive analytical features that simplify the analysis (symmetries and non-dimensional form). Bi-elliptic plane changes with the apoapsis maneuver suppressed by the use of the tidal forces are first considered. The operation of these transfers involves the choice of precise values of the longitude of the ascending node relative to the satellite-planet line, $\Omega$, and the argument of periapsis, $\omega$, which are obtained by solving an optimization problem over a Poincaré section. These surfaces are defined by the periapsis condition and repre- 
sent the orbital dynamics taken from one periapis to the next one. Comparisons with classical transfers are then given. This results in a new version of the classic optimality of bi-elliptic plane change maneuvers over one impulse plane change maneuvers ${ }^{3}$ in the case of tidally perturbed environments.

\section{Tidal transfers and Dynamics in the Hill model}

Classically, the design of orbit transfers is based on the two body problem for the underlying dynamics. In this model, the trajectory of a spacecraft is entirely determined by its five orbital elements, semi-major axis $a$, eccentricity $e$, inclination $i$, longitude of the ascending node $\Omega$ and argument of periapsis $\omega$, at one point of its orbit, until the next maneuver. In this situation, relations between two points on a given orbit are readily obtained and the problem of determinig a transfer can be viewed as a geometrical problem of the intersection of conics together with an optimization over all possible impulsive maneuvers. In particular, there is no distinction between the dynamics on the initial, final and transfer orbits. The time appears as a secondary variable (possibly as a constraint to optimize over) in the sense that if a transfer is possible at time $t_{0}$, it is also possible at time $t_{0}+T$ where $T$ represents the period of motion on the initial orbit. Also, the absolute position of the initial and final orbits relative to a given inertial frame are not generally taken into account in the formulation of the problem. Only the relative orientation of the initial orbit with respect to the final one is important. A typical example of transfer is given by the bi-elliptic plane change (or restricted three impulse plane change ${ }^{3}$ ), where a spacecraft starts in an initial low, circular orbit, a first maneuver transfers it to an ellpise where a second maneuver is performed at apoapsis, changing its orbital plane, and a final maneuver at the next periapsis retransfers the spacecraft to a low circular orbit (see figure 1).

When perturbations occur, however, this state of affairs changes. Orbital elements are now a function of time and there may be no definite analytical relationship between two points on a given orbit. The situation is not so bad for small perturbations where averaging theory gives a good approximation of the secular changes of the orbital elements over one orbit. Since by assumption these perturbations are small, variations over one orbit remain small and the two body approximation retains its value and gives useful estimates of the $\Delta V$ required for a given maneuver. This is, for example, the case for orbiters moving in a $J_{2}$ perturbed gravitational field. Moreover, in this case, the perturbations decrease as the apoapsis of the transfer orbits are raised, justifying again the two body assumption. Still, even in this slightly perturbed environment, $\omega$ and $\Omega$ are shifted during each orbit and the absolute orientation of the initial and final orbit at a given epoch enters into the problem.

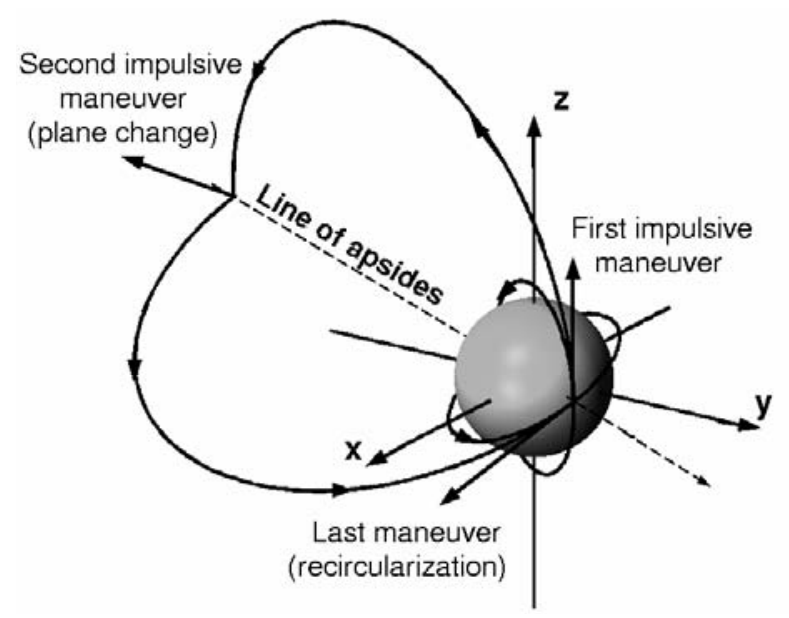

Figure 1: Geometry of bi-elliptic plane changes

In the case of a planetary satellite, the tidal perturbations may be strong enough to cause a spacecraft to impact the surface of the satellite over a single orbit. More precisely, assuming that a spacecraft is in a low circular orbit, and that a $\Delta V$ maneuver is performed to place the spacecraft onto an eccentric orbit, performing the maneuver at time $t_{0}$ or $t_{0}+T$ may result in an impact or escape from the satellite before the spacecraft has reached the next periapsis ${ }^{2}$. Unlike the $J_{2}$ perturbations, tidal forces become more prominent as one gets further from the attracting body. Dynamics close to the satellite and on the transfer orbits now have very different characteristics, and the orientation of the initial and final orbits (or equivalently the timing of the maneuvers) are of prime importance. More precisely, the initial value of $\omega$ and $\Omega$ now control, in some way, the changes that occur during a transfer orbit, and a careful choice of these variables allows us to use the tidal forces to generate the plane change without any cost (see figure 5).

After presenting the model used to characterize the tidal effects in the next subsection, the assumptions and dynamics that make such transfers possible are presented.

\section{The Hill model}

This model was first derived by $\mathrm{Hill}^{4}$ to explain the motion of the moon around the Earth, and is a useful model to characterize the motion of two close masses perturbed by a larger, massive object. In particular, this model describes the dynamics of a spacecraft in the environment 
of a planetary satellite, or even in a large orbit about a planet.

This model is given by the following set of differential equations:

$$
\begin{aligned}
\ddot{x}-2 N \dot{y} & =-\frac{\mu}{r^{3}} x+3 N^{2} x \\
\ddot{y}+2 N \dot{x} & =-\frac{\mu}{r^{3}} y \\
\ddot{z} & =-\frac{\mu}{r^{3}} z-N^{2} z
\end{aligned}
$$

where $(x, y, z)$ are the cartesian coordinates of a rotating frame (see Figure 2), $r=\sqrt{x^{2}+y^{2}+z^{2}}$ is the magnitude of the radius vector, $N$ is the angular velocity of the satelite (planet) around the planet (sun), and $\mu$ is its gravitational parameter.

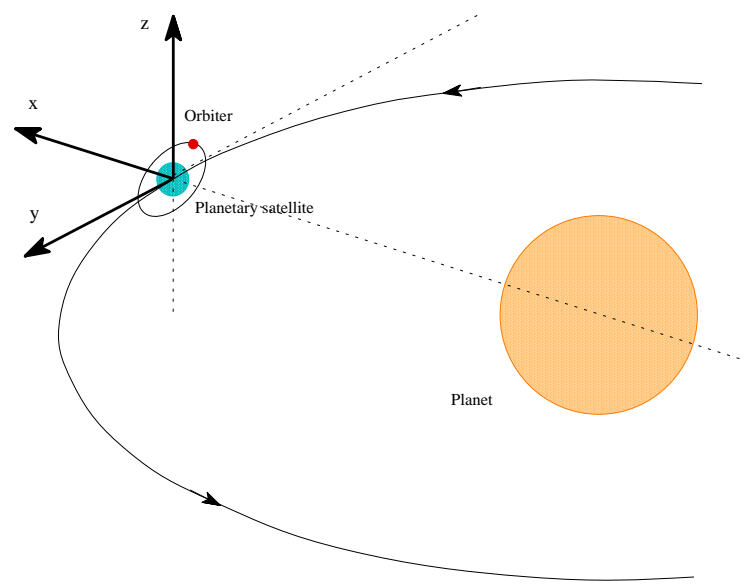

Figure 2: Geometry of the Hill problem

These equations can be made nondimensional and parameterless by setting the length and time scales to be $l=\left(\mu / N^{2}\right)^{1 / 3}$ and $\tau=1 / N$ respectively. The resulting equations are obtained by setting $\mu=1$ and $N=1$ in the previous set of equations. Therefore all the analysis performed on the nondimensional system can be scaled to any physical system by setting the proper length and time scale. This feature makes the Hill model an attractive dynamical model. Table 1 gives the length and time scales of a few planetary satellites of the solar system and of Mercury (as perturbed by the Sun).

Table 1: Physical parameters for Mercury, Europa, Titan, Miranda and Triton ${ }^{5}$

\begin{tabular}{lrccc}
\hline \hline \multicolumn{2}{c}{$\begin{array}{c}\text { Primary } \\
\text { (perturbation) }\end{array}$} & $\begin{array}{c}\text { Length scale } \\
(\mathrm{km})\end{array}$ & $\begin{array}{c}\text { Time scale } \\
(\mathrm{hr})\end{array}$ & $\begin{array}{c}\text { Normalized } \\
\text { radius }\end{array}$ \\
\hline Mercury & (sun) & 318,272 & 336.00 & 0.007 \\
Europa & (Jupiter) & 19,692 & 13.56 & 0.079 \\
Titan & (Saturn) & 75,576 & 60.90 & 0.034 \\
Miranda & (Uranus) & 1,214 & 5.39 & 0.199 \\
Triton (Neptune) & 38,406 & 22.44 & 0.045 \\
\hline \hline
\end{tabular}

\section{Dynamics close to the primary}

Note that $l$ and $\tau$ give the length and time scale at which the non-Keplerian phenomena of the dynamics become important. For instance, the libration points $L_{1}$ and $L_{2}$ are situated on the $x$-axis at a distance of $(1 / 3)^{1 / 3} l \simeq$ $0.69 l$ from the center of mass and $2 \pi \tau$ is the period of motion of the satellite (planet) around the massive planet (sun). This shows that for small values of radius and short time scales (as compared to $l$ and $\tau$ ), the dynamics become close to Keplerian and motion is only slightly disturbed. This is in accordance with our physical intuition of a tightly bound trajectory (the disturbung planet is far away and its gravitational pull is small when compared to the primary) or the notion of a sphere of influence in interplanetary trajectories. Note also that for $N=0$ the Hill model reduces to the two body problem.

In such a realm of motion, tidal perturbations can be averaged over as has been done in Scheeres et $\mathrm{al}^{1}$. In that paper, a double averaging has been performed, since one order of magnitude appears between the motion of the spacecraft around the satellite and the motion of the satellite around the planet (i.e., the motion is considered for low nearly circular orbits of normalized radius less than 0.215 ). The results of the analysis shows that, even though the tidal perturbations are small, they can generate instabilities that can cause a spacecraft to impact the surface of the satellite after a few weeks or months for the case of Europa. These instabilities appear only for certain ranges of the inclination (near polar orbits, $39.23^{\circ}<i<140.77^{\circ}$ ) and are characterized by an exponential growth of the eccentricity. However, the time scale for such an exponential growth is large when compared to the orbital motion of the spacecraft, and is an order of magnitude larger than the period of Europa about the planet (as a result of the double averaging). The semimajor axis and inclination remain constant on average (at first order in $e$ ), which means that, starting from an initial quasi-circular orbit of eccentricity 0.001 , the eccentricity will be less than 0.01 for a number of revolutions of Europa around the planet. In other words, as concerns orbital transfer maneuvers, one can assume that a spacecraft is in a low, circular orbit with a precessing ascending node. The orbital elements $\Omega$ and $\omega$ of the transfer maneuver, determined by the first maneuver, can then be set close enough to any prescribed value by a simple timing of the maneuver.

Table 2 gives some characteristics of these instabilities for the few celestial bodies mentioned in the previous table, together with a bound on the positioning error for $\omega$ and $\Omega$ for a time span of one satellite period. More precisely, the characteristic instability time $\tau_{S}$ represents the inverse of the exponential characteristic of the eccentricity growth $\left(e(t) \simeq e^{\lambda t}\right.$ and $\left.\tau_{S}=1 / \lambda\right)$ for a polar orbit. 
The eccentricity will grow by an order of magnitude after $\ln (10) \simeq 2.302 \ldots$ characteristic times. Finally, assuming that the mean motion of the spacecraft is $n$ and the mean motion of the primary is $N$, the simple rotation of the frame implies that, given some nominal value $\Omega_{0}$ of the longitude of the ascending node, the spacecraft can reach any value of mean anomaly within one revolution around the primary and a shift in $\Omega$ by $\pm 180 N / n$. Note that this positional accuracy will improve orbit after orbit.

Table 2: Parameters for the dynamics close to Mercury, Europa, Titan, Miranda and Triton ${ }^{1}$

\begin{tabular}{cccc}
\hline \hline Primary & $\begin{array}{c}\text { Characteristic } \\
\text { instability } \\
\text { time } \tau_{S} \text { (days) }\end{array}$ & $\begin{array}{c}\text { Mean } \\
\text { motion } \\
\text { ratio } N / n\end{array}$ & $\begin{array}{c}\text { Positioning } \\
\text { error on } \Omega \\
\text { (in degree) }\end{array}$ \\
\hline Mercury & $>30$ years & 0.00067 & 0.12 \\
Europa & 13.66 & 0.0225 & 4.05 \\
Titan & 220.28 & 0.00627 & 1.12 \\
Miranda & 1.36 & 0.0893 & 16.07 \\
Triton & 31.20 & 0.0163 & 2.93 \\
\hline \hline
\end{tabular}

Outside this instability region, the eccentricity is shown to oscillate around its inital value and, once again, one can assume the spacecraft to be in a circular orbit over a number of orbits. These assumptions are by no means necessary for the practical application of the transfers described below, but ease the understanding of the geometry of the maneuver and the comparisons to be made.

\section{Orbital dynamics}

As mentioned earlier, the idea of our tidal transfers is to place the spacecraft into a highly eccentric transfer trajectory (to raise the apoapsis radius) so that the large tidal forces can perform plane change maneuvers without any $\Delta V$ costs (at apoapsis). This implies that the spacecraft will start from one periapsis and follow the transfer trajectory to the next periapsis (see figure 5). Such motion is then fully characterized by the change in orbital elements from one periapsis to the next one. These orbital dynamics are, in spirit, the same as a Poincaré section used to investigate the dynamics close to a periodic orbit. Here, the sections are defined by the periapsis condition $\dot{r}=0$ and $\ddot{r}>0$.

These conditions define a 5-dimensional manifold in the phase space of the system which is transversal to the flow for periapsis smaller than 0.2 in the Hill problem ${ }^{6}$ (which contains the domain of phase space in which we are interested: low initial and final circular orbits).

The initial phase space is furthermore restricted by assuming that the spacecraft is in an initially circular orbit with a given semi-major axis and inclination, and that the $\Delta V$ of the first maneuver is fixed (or equivalently, that the apoapsis radius of the transfer trajectory is fixed at periapsis). The only remaining variables are then the argument of periapsis and the longitude of the ascending node, parametrizing the surface of a torus.

The flow can then be integrated numerically to obtain a map of the dynamics from one periapsis to the next. Figure 3 gives an example of such a computation showing the change in periapsis radius, $\Delta r_{p}$, and inclination, $\Delta i$, over one iteration of the map.

These contour plots strongly indicate a symmetry in $\omega$ and $\Omega$. This is indeed the case, and one can check that the Hill equations of motion are invariant under several transformations, generated by three independant symmetries $S_{1}, S_{2}$ and $S_{3}$. If $(x, y, z, \dot{x}, \dot{y}, \dot{z}, t)$ denotes a solution of the equations of motion, then the trajectories obtained by applying the following transformations are also valid solutions:

$$
\begin{array}{lll}
(x, y, z, \dot{x}, \dot{y}, \dot{z}, t) & \stackrel{S_{1}}{\longrightarrow} & (-x, y, z, \dot{x},-\dot{y},-\dot{z},-t) \\
(x, y, z, \dot{x}, \dot{y}, \dot{z}, t) & \stackrel{S_{2}}{\longrightarrow} & (x,-y, z,-\dot{x}, \dot{y},-\dot{z},-t) \\
(x, y, z, \dot{x}, \dot{y}, \dot{z}, t) & \stackrel{S_{3}}{\longrightarrow} & (x, y,-z, \dot{x}, \dot{y},-\dot{z}, t)
\end{array}
$$

The composition of these three symmetries yield other symmetries, notably the composition of (1) and (2) yield:

$(x, y, z, \dot{x}, \dot{y}, \dot{z}, t) \quad \stackrel{S_{4}}{\longrightarrow} \quad(-x,-y, z,-\dot{x},-\dot{y}, \dot{z}, t)$

This last symmetry corresponds to a symmetry about the origin in the $(x, y)$-plane, which is equivalent to a symmetry of motion for shifts in $\omega$ of the form $\omega+m \pi$, $m=0, \pm 1, \ldots$. Also the $S_{3}$ symmetry (reflection about the $(x, y)$-plane) is equivalent to a symmetry in $\Omega$ of the form $\Omega \rightarrow \Omega+m \pi$.

Therefore all the dynamics of the transfer trajectories can be represented on the region $\left[0,180^{\circ}\right] \times\left[0,180^{\circ}\right]$ of the $(\omega, \Omega)$-space.

Another interesting feature of these contour plots is the presence of zero lines. That is, there exists some values of argument of periapsis and longitude of the ascending node for which the change of periapsis radius from one periapsis to the next one is zero. This is precisely one of the ingredients needed for the case of a plane change transfer.

As apoapsis is raised (i.e., the initial $\Delta V$ is incremented), tidal perturbations become stronger and can cause the spacecraft to escape from the planetary satellite (planet). Figure 4 shows two examples of such contour plots with escape regions. It should be noticed that the topology of the zero curves has changed. There is now only one zero curve and it does not fill the whole $\Omega$-space. This means that there are some time spans over which no transfer maneuvers can be performed, whatever value of $\omega$ is chosen. However, at $\Omega=0$, the $\Delta r_{p}=0$ lines still cross the $\omega$-axis at exactly two distinct points. These observations will be assumed in the computation of an optimal plane change in the next section. They are, at this point, conjectures checked only by numerical 


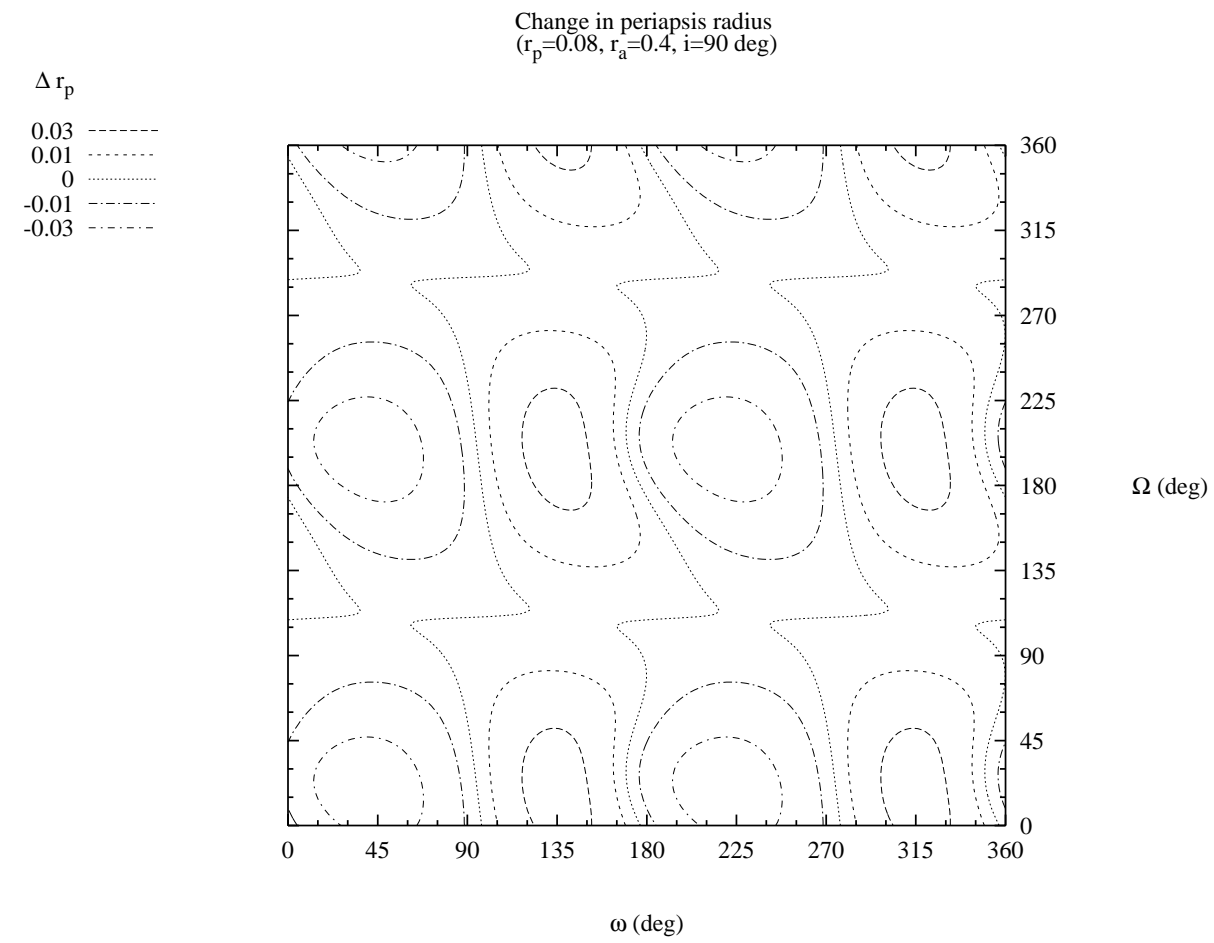

$\Delta \mathrm{i}(\mathrm{deg})$
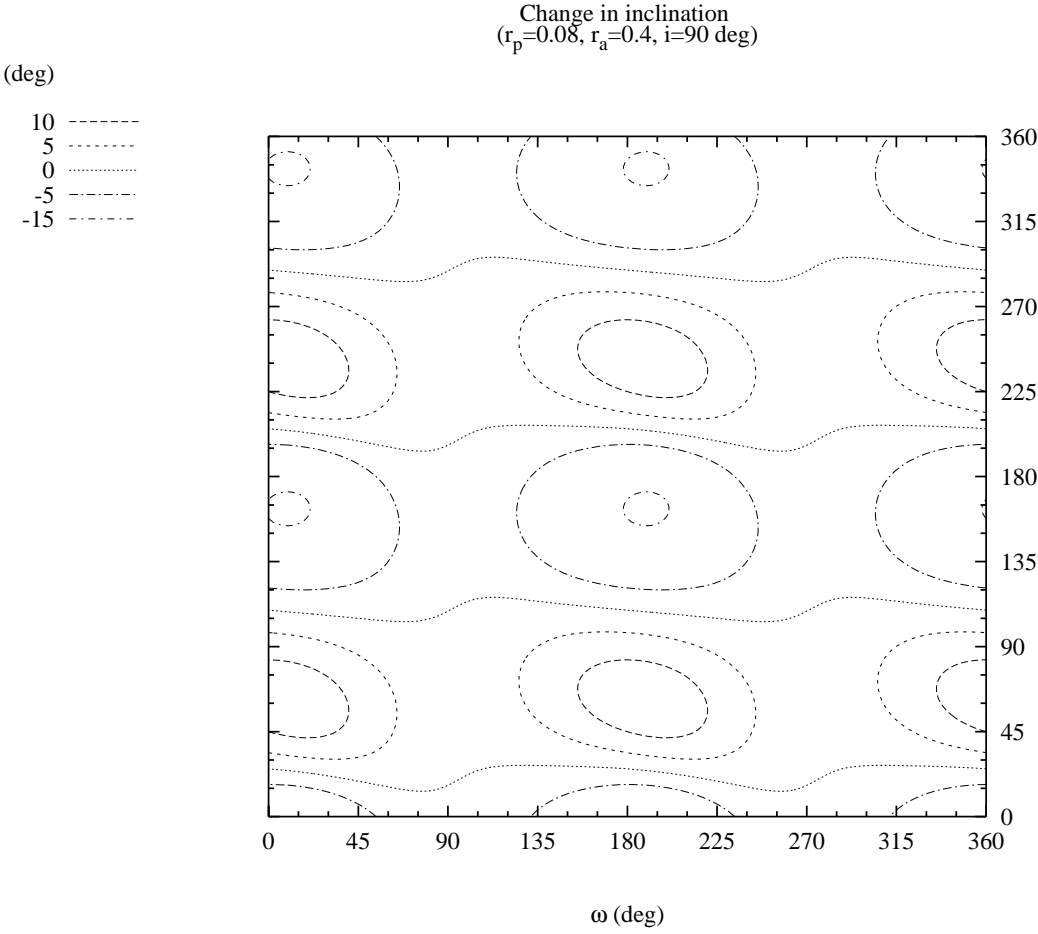

Figure 3: Contour plots for $\Delta r_{p}$ and $\Delta i$ with $r_{p}=0.08, r_{a}=0.4$ and $i=90^{\circ}$ ( $r_{p}$ represents the periapsis radius and $r_{a}$ the apoapsis radius) 
Change in periapsis radius

$\left(r_{p}=0.08, r_{a}=0.6, i=90 \mathrm{deg}\right)$

$\Delta \mathrm{r}_{\mathrm{p}}$
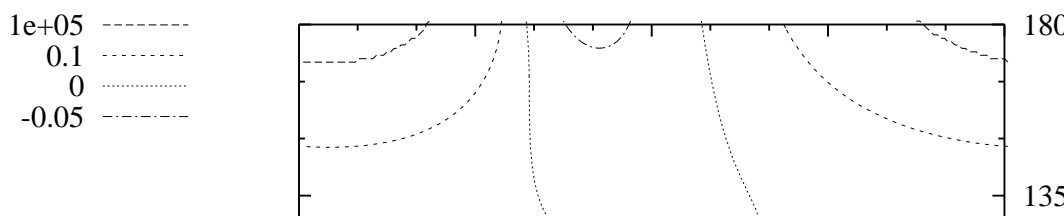

135
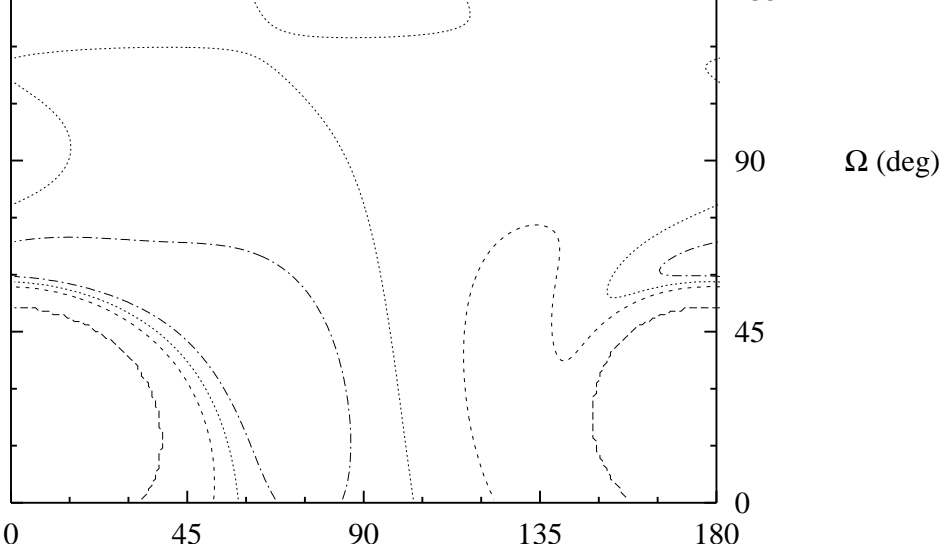

45

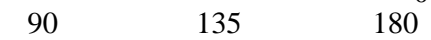

$\omega(\operatorname{deg})$

$\Delta \mathrm{i}(\mathrm{deg})$
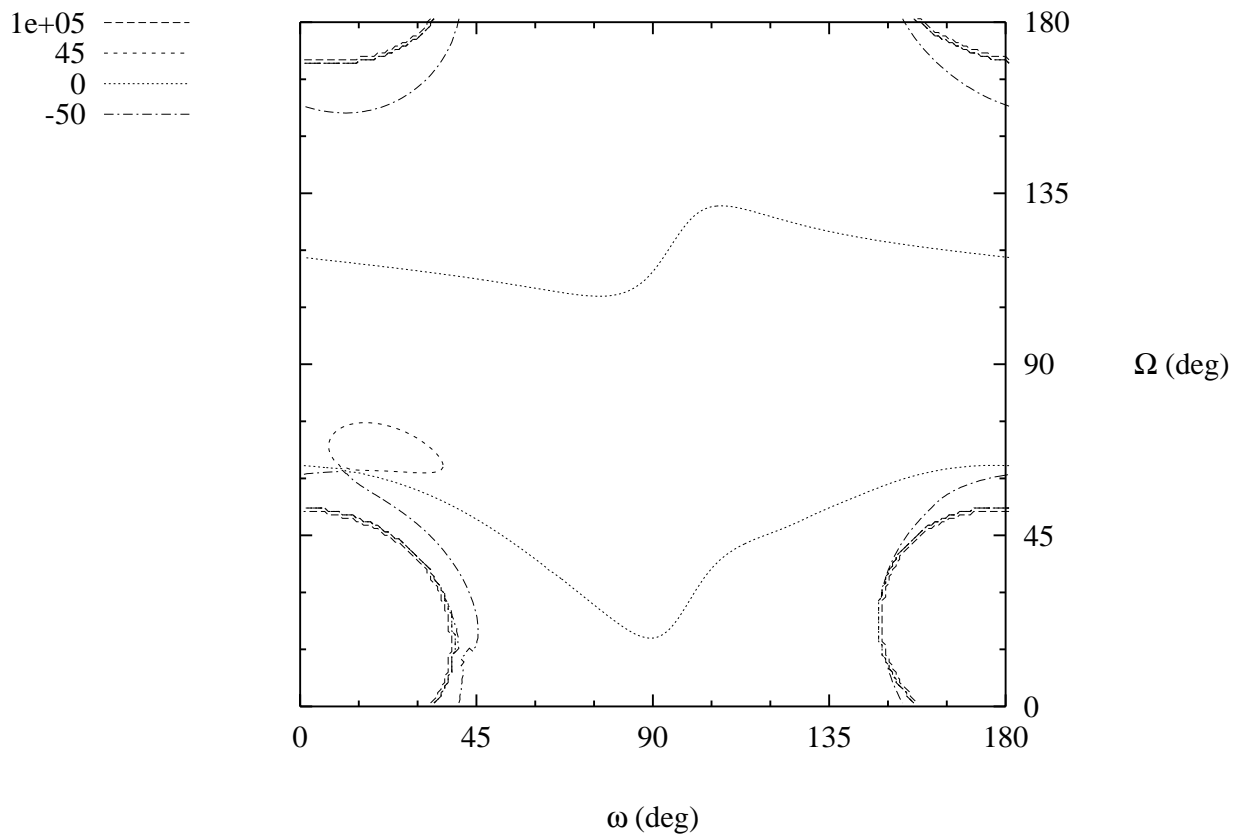

Figure 4: Contour plots for $\Delta r_{p}$ and $\Delta i$ with $r_{p}=0.08, r_{a}=0.6$ and $i=90^{\circ}$

(The contours with value $+1 e 05$ delineate the escape regions) 
computations of several contour plots at different initial values.

It can also be observed that as the inclination changes from 0 to $180^{\circ}$, the orbital element changes over one orbit get larger around $90^{\circ}$ and, at the same time, the homotopy type of the zero curves changes.

Finally, one can see on these plots that the values of $\omega$ and $\Omega$ have a very strong influence on the dynamics of the transfer orbits, as was already noticed ${ }^{2}$. A change by a few tens of degrees in one of these variable can lead to an escape or impact, all other elements remaining equal.

\section{Tidally driven plane changes}

In the previous section, we have shown that tidal perturbations can produce large changes over a single orbit, allowing the use of these forces as a control by choosing $\omega$ and $\Omega$ of the transfer orbit. In this section, we specialize the analysis to plane change maneuvers where the criteria to be met are a zero change in periapsis radius while achieving a maximum plane change over a single transfer trajectory. Inspection of the previous contour plots indicates the possibility of transfers which correspond to a large change in inclination over the $\Delta r_{p}$ zero lines. After briefly reviewing the geometry of the plane change maneuvers analysed in this paper, a numerical exploration of the possibilities of such transfers is dicussed. Maximum plane changes as a function of apoapsis radius are obtained by solving an optimization problem under constraint $\left(\Delta r_{p}=0\right)$, and comparisons with the classical one impulse maneuvers are given.

\section{Geometry of the transfers}

As noted in the introduction, the tidally driven plane changes considered in this paper are analogous to bielliptic plane changes, with the apoapsis maneuver replaced by the "cost free" action of the tidal forces. More precisely, consider a spacecraft initially in a low circular orbit. A first tangential burn places the spacecraft into a highly eccentric orbit. This first maneuver determines the elements of the transfer trajectory at periapsis. In particular, the argument of periapsis and the longitude of the ascending node are directly determined by the time at which the maneuver is performed. One can view the spacecraft moving on its initial circular orbit as describing a line (parametrized by time) on the $(\omega, \Omega)$ torus space of the previous contour plots. The value of $\omega$ and $\Omega$ at a point of this line would correspond to the value of the argument of periapsis and longitude of the ascending node of the transfer trajectory if the maneuver were performed at that point $(\Omega$ being measured with respect to the $x$-axis* at the time of the maneuver). As noticed earlier (subsection "Dynamics close to the primary") proper timing of the maneuver allows us to choose the value of these two variables with a fair accuracy.

After this first maneuver, the tidal forces cause changes in the orbital elements as the spacecraft completes one orbit. At the next periapsis, a plane change has occured while the careful choice of $\omega$ and $\Omega$ ensured that no net change in periapsis radius occured. A second tangential maneuver is then used to place the spacecraft in its final, low altitude circular orbit (see figure 5). Note that, unlike the case of the bi-elliptic plane change maneuvers, the magnitude of this recircularizing burn is not, in general, equal to the magnitude of the first maneuver.

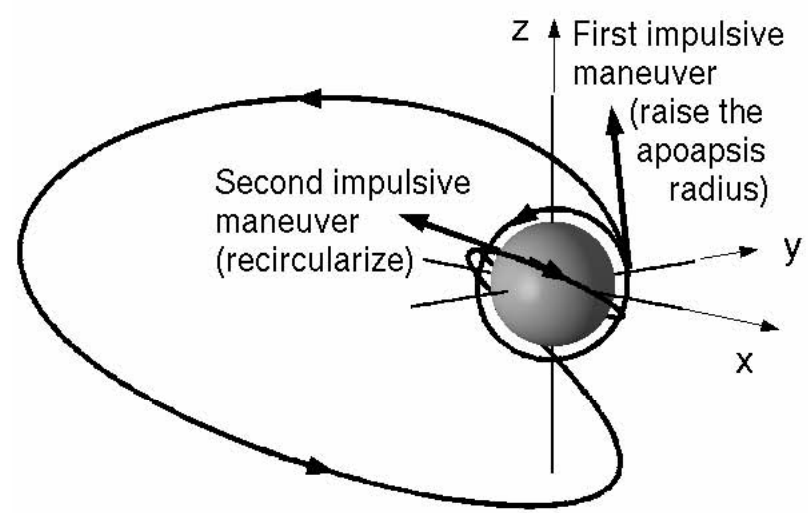

Figure 5: Geometry of tidally driven plane changes

\section{Numerical solution for the maximum and minimum plane change}

In order to choose the values of $\omega$ and $\Omega$ that yield a desired plane change, the maximum and minimum values of plane change realizable for a given value of initial inclination, periapsis and apoapsis radii have been solved. This is realized numerically by first searching the $\Delta r_{p}$ zero lines on the torus $\left[0,180^{\circ}\right] \times\left[0,180^{\circ}\right]$ and then looking for the maximum plane change on these zero lines.

The program written assumes that there is, at most, two different $\Delta r_{p}=0$ lines in this space for low inclination regions (where escape regions can exist), and at most 4 different zero lines crossing the $\omega$-axis, for regions with larger inclination (above $130^{\circ}$ ). Future developements of the programs should relax these assumptions.

A first search is hence performed on this axis to determine these intersections. More precisely, it is assumed that the zero lines are separated from each other and from the escape regions by at least $1^{\circ}$. With these assumptions, the intersection is computed to an accuracy of $5.7 \times 10^{-7}$ degrees. It should be noted that no errors

\footnotetext{
* that is, the planet-satellite or Sun-planet line
} 
have been generated during the execution of these runs and that, in most of the cases considered, two intersections have been found. Four intersections can however occur for large values of apoapsis radius, where plane changes of $-180^{\circ}$ can occur. As noticed earlier, the homotopy type of these zero lines can vary.

After this first search, the zero lines are tracked by moving, step by step, along the tangent of these zero lines. Again it is assumed that no escape region lies within $5.7 \times 10^{-5}$ degrees of these lines. This has always been the case in the domain of phase space considered. The maximum step size used to perform this tracking is $1^{o}$ and a search for the maximum and minimum $\Delta i$ is then performed over all these points. This means that the determination of the global maximum of plane change is done within this limit.

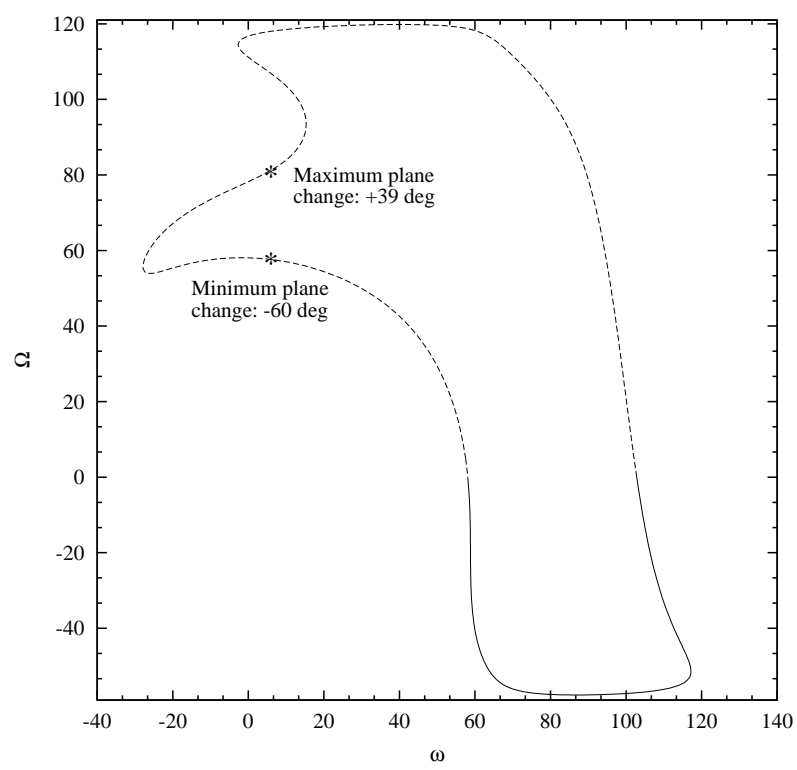

Figure 6: $\Delta r_{p}=0$ lines for $r_{p}=0.08, r_{a}=0.6$ and $i=90^{\circ}$

Figure 6 gives an example of such a computation where the zero lines have been computed for the same initial values as in figure 4 . The position of the maximum and minimum of plane change is indicated by a small label.

Once the maximum (or minimum) has been found, the accuracy is improved up to $5.7 \times 10^{-5}$ degrees, that is, the positional accuracy of the local maximum is $5.7 \times 10^{-5}$ degrees. Note that all the integration performed use a Runge-Kutta-Felhberg integrator of order $7-8$, with an accuracy of $10^{-10}$.

Finally, we should note that the dynamics considered here are smooth (or, at least, continuous), so that, when the maximum and minimum plane change are located on the same $\Delta r_{p}$ zero line, all the values of plane change between these two extrema are possible. In particular, zero plane changes are possibles. This is the case for example in figure 6. This is not true, however, for the cases of $-180^{\circ}$ plane changes indicated in the next subsection. In these cases, indeed, the maximum and minimum plane changes are located on two different, separated zero lines.

\section{Variations with the initial elements}

Once this computation has been performed for a given set of values of periapsis radius, apoapsis radius, and inclination, a loop is performed to cover a wide range of cases. Figures 7 to $10^{\dagger}$ give the result of such computations when, respectively, the initial inclination, apoapsis radius and periapsis radius are varied. Each plot has been computed with at least 90 points for the variable being varied.

Two distinct behaviors of the variations of the minimum plane change are obtained when inclination varies, according to the initial values of periapsis radius, $r_{p}$, and apoapsis radius, $r_{a}$.

For the lower values of apoapsis radius (at fixed $r_{p}$ ), the minimum plane change obtained for the inclinations of $0^{\circ}$ and $180^{\circ}$ is zero. In this case, the changes in orbital elements become larger around an inclination of $90^{\circ}$ as can be seen on figure 7 for the change in inclination.

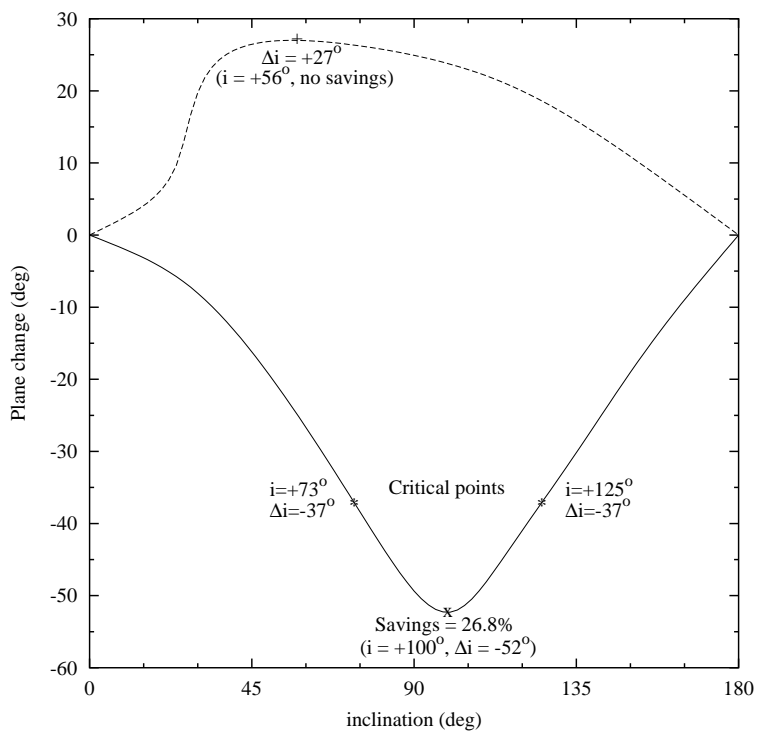

Figure 7: Optimal plane change values for $r_{p}=0.08$, $r_{a}=0.5$ as a function of inclination

For higher values of apoapsis radius, a bifurcation occurs, so that, at the inclination of $180^{\circ}$, a minimum plane change of $-180^{\circ}$ is obtained, as can be seen on figure 8. Note that this result is consistent with the invariance

\footnotetext{
${ }^{\dagger}$ Tags on these graphs are explained in the next subsection
} 
of the $(x, y)$-plane in the Hill model. A plane change of $-180^{\circ}$ at an incliantion of $180^{\circ}$ corresponds, indeed, to a change in the direction of motion relative to the primary (change from retrograde to prograde orbit), and does not involve any $z$-component of the velocity vector. The motion remains planar during such a transfer.

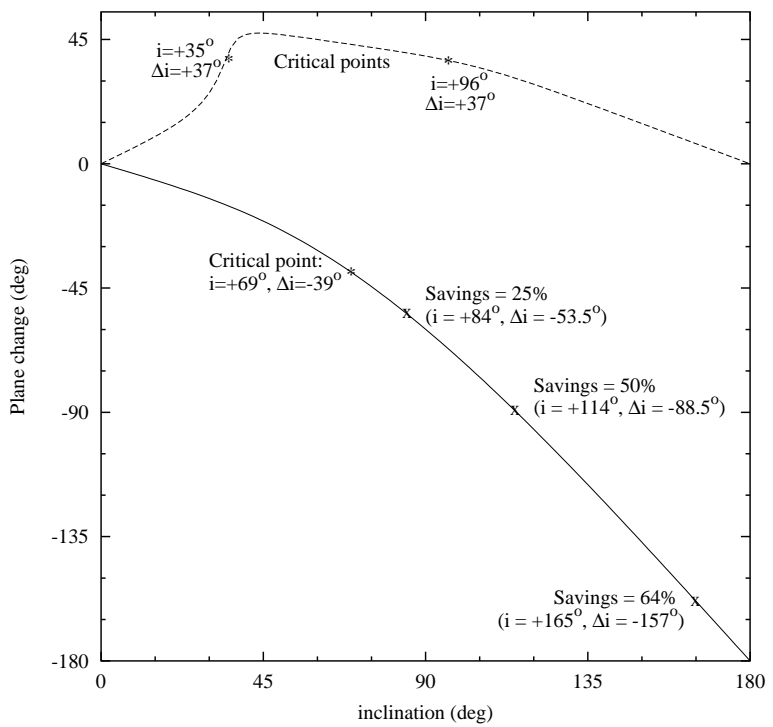

Figure 8: Optimal plane change values for $r_{p}=0.08$, $r_{a}=0.6$ as a function of inclination

For positive plane changes, no bifurcation occurs and zero plane changes are obtained at the inclinations of $0^{\circ}$ and $180^{\circ}$. Hence, we clearly see that the dynamics are not symmetric for positive and negative plane changes. Positive plane changes are larger only for lower inclinations $\left(\sim 45^{\circ}-60^{\circ}\right)$ and the overall maximum plane change is smaller than the overall minimum (i.e., taken over all the range of inclination).

In the rest of the paper, an inclination of $90^{\circ}$ has been assumed as a representative inclination for the study of the variations of plane change with apoapsis radius and periapsis radius. Such an inclination corresponds, indeed, to the mean inclination over the unstable region for planetary satellite orbiters (see subsection "Dynamics close to the primary"), and gives a good picture of the dynamics considered.

As the initial apoapsis radius is raised the plane changes obtained become larger, as expected (see figure 9). Here again, the dynamics are not symmetric for the maximum and minimum plane change, positive plane changes remaining smaller than the negative plane changes. However, in both cases, large plane changes can be obtained and for initial apoapsis radius larger than 0.645 (with $r_{p}=0.08$ and $i=90^{\circ}$ ), both positive and negative plane changes allow us to transfer an unstable polar orbiter back to a stable region over a single transfer trajectory. For such values of apoapsis radius, both positive and negative plane changes are, indeed, larger than $45^{\circ}$.

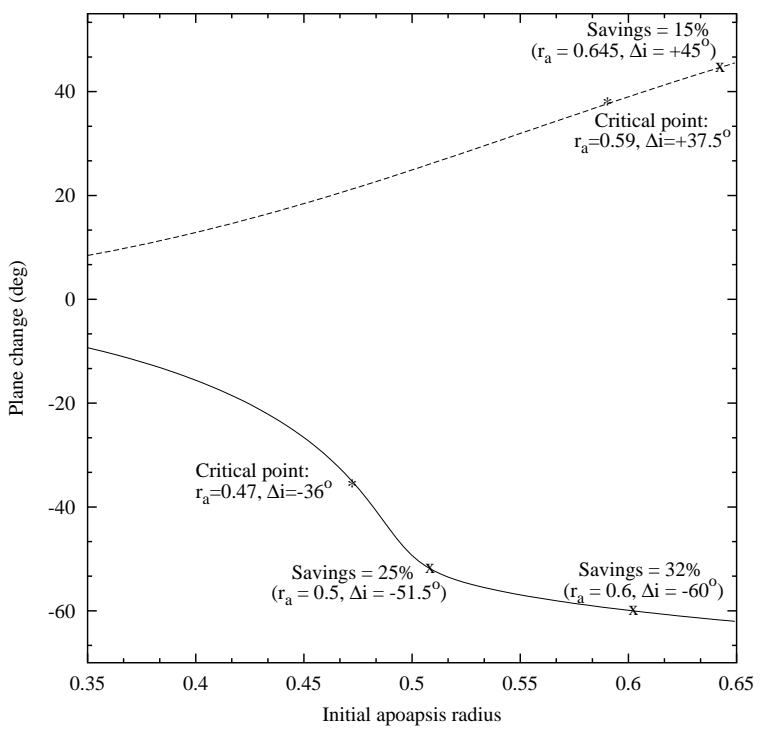

Figure 9: Optimal plane change values for $r_{p}=0.08$, $i=90^{\circ}$ as a function of apoapsis radius

At fixed apoapsis radius, a decrease in the initial periapsis radius results in the amplification of the tidal effects. As a consequence, larger plane changes can be obtained, as shown on figure 10. Note that, even though the Hill problem has a similarity property, i.e., it can be made nondimensional so that each system modeled by the Hill equation of motion can be mapped into one another by a simple scaling, it is not true that the dynamics are similar at different values of the normalized radius. In particular, an increase in $r_{p}$ with a fixed ratio $r_{p} / r_{a}$ does not mean that the plane changes realizable are the same. For instance, at $r_{p}=0.08$ and $r_{a}=0.4$, the minimum plane change is smaller (in absolute value) than $-20^{\circ}$, whereas for $r_{p}=0.1$ and $r_{a}=0.5$, the minimum plane change achievable is larger than $-40^{\circ}$. That is, as the periapsis radius increases with fixed $r_{p} / r_{a}$ ratio, the extrema of plane change achievable increase. These dynamics are also not linear in the sense that they do not depend solely on the difference between $r_{a}$ and $r_{p}$, as can be easily checked on the previous graphs.

Finally, it should be noticed that tidally driven plane changes of more than $60^{\circ}$ (both positive and negative) are possible for Earth orbiters (the normalized radius of the Earth is $\sim 0.003$ ), allowing us to compare tidal transfers 
to parabolic plane change maneuvers.

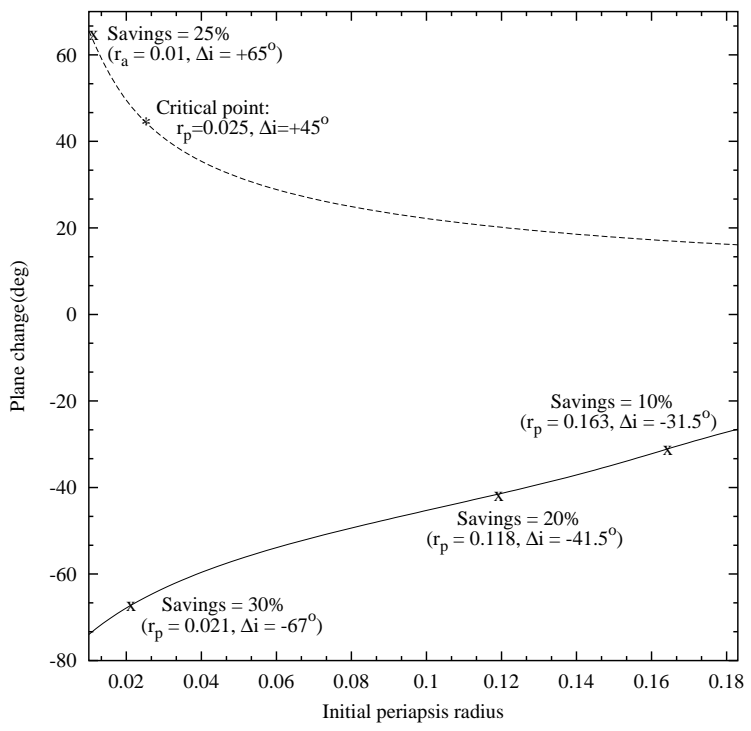

Figure 10: Optimal plane change values for $r_{a}=0.5$, $i=90^{\circ}$ as a function of periapsis radius

\section{Comparison with classical transfers and optimality}

Classical results ${ }^{3}$ on plane change maneuvers indicate that for low plane changes, one impulse maneuvers are less expensive than bi-elliptic transfers (see figure 1 for the geometry of bi-elliptic plane change maneuvers). The $\Delta V$ cost required to perform such a maneuver is given by:

$$
\Delta V_{0}=2 V_{l c} \sin (\Delta i / 2)
$$

where $V_{l c}=\sqrt{1 / r}$ is the local circular velocity speed at the time of the maneuver.

From these formulas, we can see that the cost associated with a single impulse maneuver decreases as we get further from the attracting body. As the required plane change becomes larger, it then becomes cheaper to first raise the apoapsis radius before performing the plane change maneuver. It can be shown that bi-elliptic transfers become more optimal than a direct one impulse plane change for changes in inclination, $\Delta i$, greater than $38.94^{\circ}$. In such a case, the classical optimal $\Delta V$ is given by:

$$
\Delta V=2 V_{l c}\left[\sqrt{\frac{2 \rho}{1+\rho}}\left(1+\frac{\sin (\Delta i / 2)}{\rho}\right)-1\right]
$$

where $\rho=1 /(\csc (\Delta i / 2)-2)$ is the ratio of the apoapsis radius of the transfer ellipse to the radius of the initial circular orbit.
As the desired plane change $\Delta i$ increases, we can see from the previous formulas that the optimal value of apoapsis radius of the bi-elliptic transfer increases to reach infinity at $\Delta i=60^{\circ}$. That is, for a plane change larger than $60^{\circ}$, parabolic plane change maneuvers are the classical optimal solution. In such a case the total $\Delta V$ is given by:

$$
\Delta V=2 V_{l c}[\sqrt{2}-1]
$$

As noticed earlier, in strongly perturbed tidal environments, raising the apoapsis radius cannot be done arbitrarily without inducing changes in other orbital elements. For low apoapsis radius, the tidal changes remain very small, however, and the classical approach is still valid. This is the case, for example, for plane changes performed in the Earth environment where the optimal apoapsis radius of the bi-elliptic transfer remains small in the nondimensional scale of the Hill problem. As the plane change to be performed increases, however, this approach can not be used without taking the tidal forces into consideration. That is, at some point below $\Delta i=60^{\circ}$, tidal effects can be used to reduce the cost of plane changes.

A $\Delta V$ comparison between the optimal classical transfers (given by equations 4 to 6) and tidally driven plane changes has been performed. The tags on the graphs shown in figures 7 to 10 indicate some values of the savings associated with tidally driven plane changes when compared to one impulse maneuvers. It should be noticed that savings larger than 25 percent are obtained for large plane change maneuvers $\left(\sim 60^{\circ}\right)$. The critical points also indicated on these figures, show the values of the initial elements and plane change for which the transition of optimality between one impulse maneuvers and tidally driven plane changes occurs. That is, for plane changes larger than the critical values, tidally driven plane changes are more optimal than one impulse maneuvers. Note that, in fact, except for the critical point shown on figure 10 , the transition generally occurs before the transition considered in the classical setting $\left(38.94^{\circ}\right)$, so that tidally driven plane changes become more optimal than the classical approach at these critical points. For the case of figure 10, it can be checked that the transition of optimality (as compared to the optimal classical approach) is reached at $\Delta i=46^{\circ}$.

Note that for plane changes larger than $60^{\circ}$, tidally driven plane changes still remain more optimal than the classical optimum of parabolic plane changes. Savings on the order of $5-10 \%$ are obtained in the case of figure 10 and more than $15 \%$ in the case of figure 9 . These values give an idea of what should be achievable for the case of Earth orbiters, since the normalized radius of the Earth is $\sim 0.003$. Also, we should note the large savings made for the case of change of direction of motion (plane 
changes of $-180^{\circ}$ ), as shown on figure 8 . In this case, savings of more than $15 \%$ are obtained when compared to the parabolic transfers. In addition, such transfers have the advantage of remaining bounded, and in particular, the transfer time is much smaller than in the parabolic case.

All these results show that the classical optimality of bi-elliptic transfers over one impulse maneuvers for plane changes larger than $38.94^{\circ}$ must be replaced by the optimality of tidally driven plane change over the classical transfers (and in particular over the one impulse plane change maneuvers) for plane changes larger than $\sim 38^{\circ}$ (the precise critical value depending on the initial elements $r_{p}, r_{a}$, and $i$ ).

Note, however, that in the classical case, the general three impulse maneuver, that is a bi-elliptic transfer with plane changes performed at each burn, is always more optimal than one-impulse and bi-elliptic maneuvers ${ }^{3}$. A similar type of transfer should be possible in the tidally perturbed case, improving the optimality criterion obtained in this article. The analysis becomes much more involved, however, as the number of degree of freedom increases.

Finally, we should note that the results presented here can be modified to match the more general case of radius and plane change maneuvers. Instead of computing the zero lines of $\Delta r_{p}$, one has to compute the lines $\Delta r_{p}=\alpha$ where $\alpha$ represents the amount of desired radius change. Though larger savings may be possible in such cases, the order of magnitude of the savings obtained for the case $\Delta r_{p}=0$ should be attainable since these zero line correspond to a change of sign of the function $\Delta r_{p}(\omega, \Omega)$, so that performing the first maneuver a little ahead or after the values considered in this article, should yield a positive or negative net change in periapsis radius while keeping the values of plane change realized with zero periapsis change. This may be of interest for safety issues where a decrease in periapsis radius is generally not desirable.

\section{Conclusion}

Plane change maneuvers in tidally perturbed environments have been investigated. The natural tidal forces allow us to reduce the costs of these maneuvers by performing the desired plane changes without using an impulsive manuever during the transfer orbit. These new types of transfers (tidally driven plane changes) are based on the idea of the classic bi-elliptic plane change with the apoapsis maneuver suppressed. Their operation involves only a timing of the first burn in order to approximate some optimal values for the longitude of the ascending node (relative to the Sun-planet line) and the argument of periapsis of the transfer ellipse. These transfers have been shown to be optimal over the classical approach for plane changes larger than $\sim 38^{\circ}$, replacing, in many ways, the bi-elliptic plane change maneuvers. Savings on the order of $25 \%$ are obtained for plane changes of $\sim 60^{\circ}$, and the possibility of a reversal of the direction of motion (plane changes of $-180^{\circ}$ ) has been shown to exists using such an approach, realizing more than $15 \%$ savings when compared to parabolic transfers. Larger savings should be possible by considering a combination of both the classical methods and the approach outlined in this paper (i.e., a strategy using the tidal forces and performing plane change maneuvers at each burn). A better understanding of the topology of the change in orbital elements over one and one-half orbits is necessary to be able to evaluate such an approach.

\section{Acknowledgements}

The work described was funded by the IPN Technology Program and Outer Planets/Solar Probe Project by grants from the Jet Propulsion Laboratory, California Institute of Technology which is under contract with the National Aeronautics and Space Administration.

\section{References}

1. Scheeres, D.J., Guman, M.D., and Villac, B.F., "Stability analysis of planetary satellite orbiters: Application to the Europa Orbiter". Journal of Guidance, Control and Dynamics, 24(4), July-August 2001.

2. Villac, B.F., Scheeres, D.J., D’Amario, L. A., and Guman, M. D., "The effect of tidal forces in orbit transfers". Proceedings of the AAS/AIAA Spaceflight Mechanics Meeting at Santa Barbara, CA, 2001.

3. Chobotov, V.A., editor, Orbital Mechanics, pages 99103. AIAA Education Series, 2nd edition, 1996.

4. Hill, G.W., "Researches in the Lunar Theory".American Journal of Mathematics, 1, 1878.

5. Seidelmann, P.K., editor, Explanatory supplement to the Astronomical almanach, pages 704 - 711. University Science Books, 1992.

6. Villac, B.F., and Scheeres, D.J., "Escaping trajectories in the Hill three body problem and applications". Journal of Guidance, Control and Dynamics, in review, 2002.

American Institute for Aeronautics and Astronautics 